\title{
Vielfalt findet Stadt - \\ Aktivierung sozioökonomisch benachteiligter Menschen für den Erhalt der biologischen Vielfalt
}

Das Projekt „Entwicklung und Erprobung didaktischer Modelle zur Aktivierung benachteiligter Gruppen für den Erhalt der biologischen Vielfalt" („Vielfalt findet Stadt") der Hochschule für nachhaltige Entwicklung Eberswalde (HNEE) in Kooperation mit der Stiftung WaldWelten ist im Mai 2019 gestartet. Es wird im Bundesprogramm Biologische Vielfalt durch das Bundesamt für Naturschutz mit Mitteln des Bundesministeriums für Umwelt, Naturschutz und nukleare Sicherheit gefördert.

Die Projektarbeit richtet sich vorrangig an Kinder, Jugendliche und Erwachsene, die in sozialen Brennpunkten leben und sich bisher kaum mit Themen des Natur- und Umweltschutzes auseinandergesetzt haben. Ziel des Projektes ist es, sozioökonomisch benachteiligte Menschen durch ihre aktive Beteiligung am Naturschutz in ihrem Verantwortungsgefühl zu bestärken und ihr Engagement erlebbar zu machen. Durch eine enge Gruppenarbeit von der Ideenentwicklung bis zur gemeinsamen Umsetzung werden neben einem ganzheitlichen Verständnis für die Thematik die zwischenmenschlichen Beziehungen und das Vertrauen in die eigenen Fähigkeiten gefördert; die Zusammenarbeit schafft also Selbstvertrauen und stärkt sowohl die wahrgenommene als auch die reelle Selbstwirksamkeit. Perspektivisch soll damit eine langfristige Einbindung der Teilnehmenden in Naturschutzinitiativen sowie eine nachhaltige Wirkung als Multiplikatorinnen und Multiplikatoren im eigenen Umfeld erreicht werden.

Umweltbildnerinnen und Umweltbildner entwickeln im Rahmen des Projektes Bildungsprogramme, über die sie die Teilnehmenden erstmals in Aktivitäten und Prozessen der nachhaltigen Naturschutzarbeit, speziell zum Schutz und Erhalt der biologischen Vielfalt, einbinden. Am Anfang des Programms stehen die persönliche Naturerfahrung und die Auseinandersetzung mit Fragen der biologischen Vielfalt. Sensi- bilisiert für die Thematik setzen die Kinder und Erwachsenen anschließend in Praxisprojekten vielfältige Maßnahmen in ihrem unmittelbaren Lebensumfeld um und erarbeiten dabei Fähigkeiten im praktischen Naturschutz.

Alle im Projekt umgesetzten Maßnahmen sind unmittelbar am Erhalt und an der Förderung der biologischen Vielfalt ausgerichtet. Die Teilnehmenden werten in erster Linie städtische und stadtnahe Lebensräume auf. So legen sie Blühwiesen auf artenarmen Ruderalflächen an oder pflanzen seltene heimische Strauch- und Baumarten. Durch die Umgestaltung der Flächen schafft das Projekt nicht nur Lebensräume für Pflanzen und Tiere, sondern auch ansprechende naturnahe Aufenthaltsorte für Anwohnerinnen und Anwohner.

Die Gesamtkonzeption aller Veranstaltungen und Maßnahmen folgt von der jeweiligen vorbereitenden Planung der Umweltbildungseinheiten durch die Umweltbildnerinnen und Umweltbildner, über die partizipative Beteiligung der Teilnehmenden in gemeinsamen Beratungen bis hin zur praktischen Umsetzung der Ideen den Grundsätzen der Bildung für nachhaltige Entwicklung. Die Auseinandersetzung mit dem Thema biologische Vielfalt unter Einbeziehung von Fragen zu Ökologie, Ressourcenverbrauch oder Klimawandel soll zu einer Sensibilisierung und nachhaltigen Beteiligungsbereitschaft der Teilnehmenden beitragen. Zum Abschluss des Projekts werden alle Umweltbildungseinheiten gebündelt für Lehrende, Umweltbildner und Umweltbildnerinnen bundesweit zur Verfügung gestellt. Weitere Information können unter dem folgenden Link eingesehen werden: www.vielfalt-findet-stadt.de

Carolin Schlenther, Hochschule für nachhaltige Entwicklung Eberswalde (HNEE) doi.org/10.31244/zep.2020.01.08 